\title{
Effects of the Mixed Fermentation of Torulaspora delbrueckii and Saccharomyces cerevisiae on the Non-Volatile and Volatile Compounds and the Antioxidant Activity in Golden Dried Longan Wine
}

\author{
Kanokchan Sanoppa ${ }^{1}$, Tzou-Chi Huang ${ }^{2}$, and Ming-Chang $\mathrm{Wu}^{1}{ }^{1}$ \\ ${ }^{1}$ Department of Food Science, College of Agriculture, ${ }^{2}$ Department of Biological Science and Technology, College of Agriculture, \\ National Pingtung University of Science and Technology, Shuefu Road, Neipu, Pingtung 91201, Taiwan
}

Received: July 10, 2019 / Revised: November 7, 2019 / Accepted: November 21, 2019

\begin{abstract}
The aim of this study was to investigate the effects of Torulaspora delbrueckii and Saccharomyces cerevisiae, as pure fermenters and mixed fermenters (simultaneous and sequential culture), on the production of nonvolatiles and volatiles, and on the antioxidant activity in Golden Dried Longan juice and Golden Dried Longan wines. Alanine, arginine, glutamic acid, leucine, proline, and gamma-aminobutyric acid (GABA) were the most prominent amino acids that were found in these wines. The Golden Dried Longan Wine fermented with monocultures of S. cerevisiae and $T$. delbrueckii produced a total volatile aroma content of $393.21 \mathrm{mg} / \mathrm{l}$ and $383.20 \mathrm{mg} / \mathrm{l}$, respectively. Simultaneous culture of the two organisms produced the highest total volatile aroma content, that affected most volatile compounds including isobutanol, ethyl acetate, ethyl decanoate, ethyl heptanoate, ethyl hexanoate, ethyl pentanoate, isoamyl acetate, and isobutyl acetate. Of the four treatments, the sequential culture possessed the highest total phenolic content (5.80 mg gallic acid equivalents (GAE)/ml). In addition, the total phenolic content significantly correlated with the antioxidant activity of the Golden Dried Juice and Golden Dried Longan Wine. These results suggest that co-cultures of the two organisms used in the production of the Golden Dried Longan Wine may improve the intensity and complexity of its aroma.
\end{abstract}

Keywords: Torulaspora delbrueckii, Saccharomyces cerevisiae, simultaneous, sequential, antioxidant activity

\section{Introduction}

Longan (Dimocarpus longan Lour) is a tropical fruit grown in the Asia Pacific region, especially Thailand. Longan can be served fresh or processed as dried longan, wine, and jam. Dried fruit is a favorite product for consumers because of its unique flavor. Dried longan has been found to contain glucose, sucrose, and fructose, and also free amino acids that have been found to be associated

\section{*Corresponding author}

Tel: +886-9-32775187, Fax: +886-8-7740378

E-mail: globalizationwu@gmail.com

(c) 2020, The Korean Society for Microbiology and Biotechnology with flavor precursors in winemaking. The major volatile compounds from dried longan include ocimenes, furfural, 5-methyl furfural, isoamyl alcohol, linalool oxide, trans geraniol, benzenemethanol, ethyl hexadecanoate, 2-acetylfuran, 2-furancarboxylic acid, and 2, 5 dihydrofuran [1].

The use of non-Saccharomyces species has attracted interest for commercial winemaking as several studies have shown the potential production of a greater diversity of aromatic compounds in wine [2]. One of the nonSaccharomyces yeasts, Torulaspora delbrueckii, is already currently used in commercial winemaking [3], as this yeast has been reported to have a positive impact on the taste and aroma of wine together with a decreased 
production of undesirable compounds [4]. However, Hensen et al. [5] reported that $T$. delbrueckii had less tolerance to limited oxygen conditions compared to $S$. cerevisiae. Hence, the mixed inoculation of $S$. cerevisiae and T. delbrueckii could improve the aroma complexity and intensity of wine, including from phenylethanol, isoamyl acetate, isobutyl acetate, ethyl hexanoate, 3hydroxybutanoate, ethyl decanoate, and terpenes [6, 7].

Golden dried longan is a processed agricultural product of longan fruit, which is an important economic crop of Thailand [8]. Most research into longan wines have focused only on longan wines made from fresh longan [9-11]. In the present research, golden dried longan was chosen as a raw material for winemaking because it is considered to have a rich amount of amino acids and sugar suitable for yeast culture [1,9]. Furthermore, most studies on longan wine to date have focused on the characteristic flavor of fresh longan wine, and a few research studies have evaluated the total phenolic content and the antioxidant activity [11].

The main objective of the present research was to determine the effect of $S$. cerevisiae in association with T. delbrueckii by mono, simultaneous, and sequential cultures on the aroma and amino acids, and to analyze the functional activity (total phenolic content and the antioxidant activity as determined by DPPH, ABTS, and FRAP methods) of golden dried longan wines.

\section{Materials and Methods}

\section{Yeast strains and preparation}

S. cerevisiae strain C12 L.A. Bayanus and T. delbrueckii ATCC 20100; I AM 4383 were obtained from Blue $\mathrm{H}_{2} \mathrm{O}$ Filtration Pty Ltd. (Australia) and the Bioresource Collection and Research Center (Taiwan), respectively. The freeze-dried yeasts were propagated in a sterile nutrient broth $(0.25 \% \mathrm{w} / \mathrm{v}$ yeast extract, $2 \% \mathrm{w} / \mathrm{v}$ glucose, $0.25 \% \mathrm{w} / \mathrm{v}$ malt extract, and $0.25 \% \mathrm{w} / \mathrm{v}$ bacteriological peptone at $\mathrm{pH}$ 5.0) under static conditions at $25^{\circ} \mathrm{C}$ for up to $48 \mathrm{~h}$, and finally stored at $-80^{\circ} \mathrm{C}$ in YM medium with adding glycerol $20 \% \mathrm{v} / \mathrm{v}$ before use.

\section{Materials and fermentation conditions}

Golden dried longan was obtained from a local market at Lamphun, Thailand, which was further dried by a hot air dryer at $70^{\circ} \mathrm{C}$ for $8 \mathrm{~h}$, with the shell and seed removed before the drying. The dried longan was finally stored at $4^{\circ} \mathrm{C}$ until analysis was performed.

\section{Pretreatment and fermentation process of dried longan juice}

First, $100 \mathrm{~g}$ of golden dried longan was placed into a flask and $300 \mathrm{ml}$ of distilled water was added. The sample was homogenized to form longan juice using a blender and then passed through a filter. The longan juice was acidified to $\mathrm{pH} 3.5$ with $50 \%$ w/v DL-malic acid (Canada) and the ${ }^{\circ}$ Brix adjusted to $20.0 \%$ with pure sucrose [12].

Multiple fermentations of the sterile longan wine were conducted at $25^{\circ} \mathrm{C}$ under static conditions for 14 days: two with the monocultures and two with the two co-cultures (simultaneous and sequential). The monocultures and co-cultures were inoculated with $1 \times 10^{6} \mathrm{CFU} / \mathrm{ml}$ for both $T$. delbrueckii and S. cerevisiae. The simultaneous inoculation involved inoculating with T. delbrueckii and $S$. cerevisiae at the same time. The sequential inoculation involved inoculating with $T$. delbrueckii first, followed by inoculating with $S$. cerevisiae after $24 \mathrm{~h}$.

\section{Analysis of the amino acids}

Alanine, arginine, aspartic acid, GABA, glutamic acid, glycine, isoleucine, leucine, lysine, phenylalanine, proline, serine, threonine, tyrosine, and valine were supplied by Sigma Chemicals (USA). AccQ-Flour reagent kits containing reagent powder, borate buffer, and acetonitrile were supplied by Waters Co. Ltd. (Milford, USA).

Amino acids analysis was performed using an HPLC system (Water Alliance 2695 with heater, USA) based on the method developed by Water AccQ-Tag [13] and as used according to Zeng et al. [14]. Chromatographic separation by reversed-phase HPLC was performed using a Hypersil GOLD ${ }^{\mathrm{TM}}$ Column C18 (4.6 mm $\times 150 \mathrm{~mm}$, $3 \mu \mathrm{m}$ ) (Thermo Fisher Scientific, USA) equipped with a fluorescence detector (excitation, $250 \mathrm{~nm}$; emission, $395 \mathrm{~mm}$ ). The column thermostat was set at $37^{\circ} \mathrm{C}$. The injection volume was $5 \mu \mathrm{l}$ and the flow rate was 1.0 $\mathrm{ml} / \mathrm{min}$. The mobile phase was $10 \%$ AccQ-Tag eluent A (A), acetonitrile (B), and Milli-Q water (C).

\section{Analysis of the volatile compounds}

The volatiles were analyzed by the headspace (HS) solid-phase microextraction (SPME) method with 30/ $50 \mu \mathrm{m} \quad$ DVB/Carboxen ${ }^{\mathrm{TM}} / \mathrm{PDMS} \quad$ StableFlex $^{\mathrm{TM}}$ fiber 
(Supelco: 57348-U, Sigma Aldrich, Spain) coupled with GC-MS. GC-MS analysis was performed with an Agilent 7890B system, equipped with a DB-Wax UI column (60 $\mathrm{m} \times 0.25 \mathrm{~mm}$ coated with $0.25 \mu \mathrm{m}$ film thickness). The operation conditions were according to Liu et al. [11]. The program consisted of swirling the vial at $250 \mathrm{rpm}$ for $10 \mathrm{~min}$ at $40^{\circ} \mathrm{C}$, inserting the fiber to adsorb at $40^{\circ} \mathrm{C}$ for $10 \mathrm{~min}$ at $250 \mathrm{rpm}$, and then transferring the fiber for desorption at $230^{\circ} \mathrm{C}$ for $3 \mathrm{~min}$.

The odor activity values $(\mathrm{OAV} s)$ were calculated as the ratio between the average of the analytical concentrations and the odor thresholds.

\section{Total phenolic content}

The total phenolic contents were determined using the Folin-Ciocalteu method as adapted for wine analysis [11]. The results are expressed herein as mg gallic acid equivalents per milliliter of wine (mg GAE/ml).

\section{2,2-Diphenyl-1-Picrylhydrazyl (DPPH) radical scavenging activity}

The scavenging activity of these wines was determined by DPPH assay, also slightly modified from the procedure by Nuengchamnong and Ingkaninan [15]. First, $0.1 \mathrm{ml}$ golden longan wine was mixed with $2.9 \mathrm{ml}$ of $0.1 \mathrm{mM}$ DPPH methanolic solution, with the solution then kept in the dark at room temperature for $30 \mathrm{~min}$ before the absorbance was measured at $515 \mathrm{~nm}$ with a spectrophotometer.

\section{2,2'-azino-bis 3-ethylbenzthiazoline-6-sulfonic acid (ABTS) radical scavenging activity}

The ABTS assay was performed according to Buyuktuncel et al. [16]. Stock solutions were produced by mixing $7 \mathrm{mM}$ ABTS solution and $2.4 \mathrm{mM}$ potassium persulfate solution and were then kept for $14 \mathrm{~h}$ at room temperature in the dark. The solution was diluted by mixing $1 \mathrm{ml}$ ABTS solution with $60 \mathrm{ml}$ methanol to obtain an absorbance of $0.7 \pm 0.02$ as measured at $734 \mathrm{~nm}$ using a spectrophotometer. Then, $0.1 \mathrm{ml}$ of golden longan wine was added to $2.4 \mathrm{ml}$ ABTS solution. After a further $6 \mathrm{~min}$, the absorbance was measured.

\section{Ferric Reducing Antioxidant Power (FRAP)}

The FRAP assay was performed based on a previously published method by Buyuktuncel et al. [16]. The stock solution was prepared by mixing $300 \mathrm{mM}$ acetate buffer, $10 \mathrm{mM}$ 2,4,6-tripyridyl-s-triazine (TPTZ) in $40 \mathrm{mM} \mathrm{HCl}$, and $20 \mathrm{mM} \mathrm{FeCl}_{3} \cdot 6 \mathrm{H}_{2} \mathrm{O}$ in the proportion of $10: 1: 1$ at $37^{\circ} \mathrm{C}$. The FRAP reagent was prepared fresh. Next, $15 \mathrm{ml}$ of golden longan wine was mixed with $2.85 \mathrm{ml}$ FRAP solution. Then the absorbance was measured at $593 \mathrm{~nm}$ after $30 \mathrm{~min}$.

The values of the three antioxidant activity methods are expressed herein as a trolox-equivalent antioxidant capacity (TEAC).

\section{Statistical analysis}

The results were compared by ANOVA using the SPSS 20.0 software (SPSS Inc., USA) at $p<0.05$. Principle component analysis was performed on the volatile compounds using Minitab. Correlations were computed using Pearson's correlation coefficient (r) by SPSS.

\section{Results and Discussion}

\section{Amino acids}

In total, 14 amino acids were detected in the golden dried longan wines (Table 1). The main amino acids in the juice were alanine, proline, and glutamic acid, while proline was found to be the highest in wines. Proline, alanine, glutamic acid, leucine, and GABA were revealed as the main amino acids in the four fermented wines, at concentrations over $1 \mathrm{mg} / 100 \mathrm{ml}$, similar to the result found by Liu et al. [11]. The utilization of nitrogen was the highest by the $T$. delbrueckii monoculture, followed by $S$. cerevisiae culture, and then the sequential and simultaneous cultures. Proline was the major residual amino acid in the golden dried longan wines. Similar results in lychee wines were reported by Chen and Liu [12] and Chen and Liu [17]. Proline is the least preferred nitrogen source for yeast strains and is not utilized by yeast under low oxygen conditions [18]. However, proline can be synthesized by yeast cells from glutamic acid in the cytoplasm, which consists of three enzymes, namely, $\gamma$-glutamyl kinase (the product of the PRO1 gene), $\gamma$-glutamyl phosphate reductase (the product of the PRO2 gene), and $\Delta^{1}$-pyrroline-5-carboxylate (P5C) reductase (the product of the $P R O 3$ gene) [19, 20], which also convert excess proline to glutamic acid in the mitochondria in two steps by proline oxidase (the product of the PUT1 gene) and P5C dehydrogenase (the product of 
Table 1. Amino acid content $(\mathrm{mg} / 100 \mathrm{ml})$ of golden dried longan wines.

\begin{tabular}{cccccc}
\hline \multirow{2}{*}{ Amino acids } & \multirow{2}{*}{ Juice } & \multicolumn{3}{c}{ Wines } \\
\cline { 3 - 6 } & & S. cerevisiae & T. delbrueckii & Simultaneous & Sequential \\
\hline Asp & $7.03 \pm 0.17^{\mathrm{a}}$ & $0.50 \pm 0.03^{\mathrm{c}}$ & $0.45 \pm 0.02^{\mathrm{c}}$ & $0.72 \pm 0.03^{\mathrm{b}}$ & $0.57 \pm 0.02^{\mathrm{c}}$ \\
Ser & $5.54 \pm 0.08^{\mathrm{a}}$ & $0.50 \pm 0.01^{\mathrm{cd}}$ & $0.45 \pm 0.02^{\mathrm{d}}$ & $0.56 \pm 0.05^{\mathrm{bc}}$ & $0.62 \pm 0.03^{\mathrm{b}}$ \\
Glu & $20.61 \pm 0.64^{\mathrm{a}}$ & $0.87 \pm 0.03^{\mathrm{c}}$ & $0.74 \pm 0.03^{\mathrm{c}}$ & $1.52 \pm 0.02^{\mathrm{b}}$ & $1.05 \pm 0.08^{\mathrm{bc}}$ \\
Gly & $0.91 \pm 0.04^{\mathrm{a}}$ & $0.72 \pm 0.06^{\mathrm{bc}}$ & $0.76 \pm 0.02^{\mathrm{b}}$ & $0.79 \pm 0.06^{\mathrm{b}}$ & $0.65 \pm 0.06^{\mathrm{c}}$ \\
Arg & $4.38 \pm 0.17^{\mathrm{a}}$ & $0.70 \pm 0.06^{\mathrm{c}}$ & $0.66 \pm 0.03^{\mathrm{c}}$ & $0.78 \pm 0.01^{\mathrm{bc}}$ & $0.94 \pm 0.16^{\mathrm{b}}$ \\
Thr & $5.08 \pm 0.13^{\mathrm{a}}$ & $0.35 \pm 0.10^{\mathrm{bc}}$ & $0.33 \pm 0.02^{\mathrm{c}}$ & $0.48 \pm 0.03^{\mathrm{b}}$ & $0.47 \pm 0.05^{\mathrm{bc}}$ \\
Ala & $40.95 \pm 0.70^{\mathrm{a}}$ & $1.51 \pm 0.04^{\mathrm{c}}$ & $1.43 \pm 0.06^{\mathrm{c}}$ & $2.51 \pm 0.03^{\mathrm{b}}$ & $1.65 \pm 0.15^{\mathrm{c}}$ \\
Pro & $26.13 \pm 0.84^{\mathrm{a}}$ & $21.80 \pm 1.41^{\mathrm{b}}$ & $21.92 \pm 1.59^{\mathrm{b}}$ & $19.78 \pm 0.22^{\mathrm{c}}$ & $20.51 \pm 0.17^{\mathrm{bc}}$ \\
GABA & $3.01 \pm 0.15^{\mathrm{a}}$ & $0.81 \pm 0.09^{\mathrm{c}}$ & $0.62 \pm 0.04^{\mathrm{d}}$ & $1.17 \pm 0.08^{\mathrm{b}}$ & $1.07 \pm 0.20^{\mathrm{b}}$ \\
Tyr & $0.91 \pm 0.19^{\mathrm{a}}$ & $0.42 \pm 0.03^{\mathrm{c}}$ & $0.33 \pm 0.03^{\mathrm{c}}$ & $0.65 \pm 0.02^{\mathrm{b}}$ & $0.42 \pm 0.07^{\mathrm{c}}$ \\
Val & $2.58 \pm 0.13^{\mathrm{a}}$ & $0.72 \pm 0.12^{\mathrm{bc}}$ & $0.64 \pm 0.05^{\mathrm{c}}$ & $0.81 \pm 0.03^{\mathrm{b}}$ & $0.83 \pm 0.03^{\mathrm{b}}$ \\
Ile & $0.76 \pm 0.07^{\mathrm{a}}$ & $0.32 \pm 0.08^{\mathrm{cd}}$ & $0.26 \pm 0.02^{\mathrm{d}}$ & $0.46 \pm 0.01^{\mathrm{b}}$ & $0.39 \pm 0.04^{\mathrm{bc}}$ \\
Leu & $2.90 \pm 0.08^{\mathrm{a}}$ & $1.07 \pm 0.20^{\mathrm{cd}}$ & $0.92 \pm 0.03^{\mathrm{d}}$ & $1.32 \pm 0.02^{\mathrm{b}}$ & $1.27 \pm 0.15^{\mathrm{bc}}$ \\
Phe & $N D$ & $0.77 \pm 0.11^{\mathrm{b}}$ & $0.60 \pm 0.05^{\mathrm{c}}$ & $0.96 \pm 0.10^{\mathrm{a}}$ & $0.86 \pm 0.06^{\mathrm{ab}}$ \\
\hline
\end{tabular}

Values of different superscripts in the same row are significantly different $(p<0.05)$ ND not detected

the PUT2 gene) [21, 22]. Furthermore, proline can be synthesized from arginine by two enzymes, namely, arginase (the product of the $C A R 1$ gene) and ornithine transaminase (the product of the CAR2 gene) [23, 24].

\section{Volatile compounds}

A total of 27 volatile compounds were detected in golden dried longan wines, including alcohols, ester, terpenes, and acid (Table 2). Dried longan wine cultured simultaneously using $T$. delbrueckii and $S$. cerevisiae had the highest the concentration of total volatile compounds $(471.64 \mathrm{mg} / \mathrm{l})$ due to the production of high contents of alcohols and esters, followed by sequential, $T$. delbrueckii and S. cerevisiae cultures, respectively. Similar previous studies found that a co-culture with $T$. delbrueckii and $S$. cerevisiae could improve the aroma intensity and complexity of wine. Different chemical hydrolysis rates or enzymatic hydrolysis and synthesis affect the evolution of volatile compounds [6, 7]. Alcohols were the most abundant of the volatile compounds in golden dried longan wines. Ethanol, ethylhexanol, isobutanol, isoamyl alcohol, methionol, and 2-phenylethanol were the main alcohols produced by the four fermented wines. Ethanol was the most prominent alcohol, with the $S$. cerevisiae monoculture producing the significantly highest ethanol content, followed by $T$. delbrueckii, and the simultaneous and sequential cultures, respectively. S. cerevisiae can quickly grow under low oxygen conditions and is tolerant to a high ethanol production, whereas T. delbrueckii slowed down fermentation and produced less ethanol [6, 17, 25]. On the other hand, the co-culture could improve the levels of isobutanol and isoamyl alcohol, which was similar to the results reported by Chen and Liu [17], who found that the highest concentration of isobutanol and isoamyl alcohol in lychee wines was from cultures with a co-culture compared with different yeast monocultures, which was also similar to research published by Arslan et al. [25] in Narince wines. The production of 2-phenylethanol did not show significant differences among the four fermented wines. In addition, the results found methionol in dried longan wines, which gives a cauliflower and cooked potato flavor, and its content exceeded its threshold value of $1 \mathrm{mg} / \mathrm{l}$ in the wine cultured with $T$. delbrueckii [25], while $T$. delbrueckii also increased the content of methionol in Chardonnay wines [3]. Higher alcohols, such as 2-phenylethanol, isobutanol, and isoamyl alcohol were produced via the Ehrlich pathway, which involves the utilization of amino acids. Aromatic amino and branched-chain amino acids are important precursors of 
Table 2. Volatile compounds content (mg/l) in golden dried longan wines.

\begin{tabular}{|c|c|c|c|c|c|}
\hline \multirow{2}{*}{ Compounds } & \multirow{2}{*}{$\begin{array}{l}\text { Golden dried } \\
\text { longan juice }\end{array}$} & \multicolumn{4}{|c|}{ Golden dried longan wines } \\
\hline & & S. cerevisiae & T. delbrueckii & Simultaneous & Sequential \\
\hline \multicolumn{6}{|l|}{ Alcohols } \\
\hline Ethanol & $14.38 \pm 4.82^{\mathrm{C}}$ & $160.03 \pm 3.51^{\mathrm{a}}$ & $146.76 \pm 8.24^{b}$ & $143.80 \pm 4.75^{\mathrm{b}}$ & $142.80 \pm 5.01^{b}$ \\
\hline Ethylhexanol & ND & $0.71 \pm 0.10^{b}$ & $1.14 \pm 0.13^{\mathrm{a}}$ & ND & ND \\
\hline Isobutanol & ND & $10.20 \pm 0.63^{b}$ & $7.61 \pm 1.16^{c}$ & $21.54 \pm 0.53^{\mathrm{a}}$ & $20.45 \pm 0.77^{\mathrm{a}}$ \\
\hline Isoamyl alcohol & ND & $67.14 \pm 3.09^{b}$ & $60.59 \pm 4.70^{b}$ & $97.59 \pm 6.60^{\mathrm{a}}$ & $102.41 \pm 6.88^{\mathrm{a}}$ \\
\hline Methionol & ND & $1.04 \pm 0.14^{c}$ & $1.35 \pm 0.11^{\mathrm{a}}$ & $1.29 \pm 0.06^{\mathrm{ab}}$ & $1.12 \pm 0.06^{\mathrm{bc}}$ \\
\hline 2-Phenylethanol & ND & $27.08 \pm 1.22^{\mathrm{NS}}$ & $29.73 \pm 0.69^{\mathrm{NS}}$ & $29.18 \pm 2.90^{\mathrm{NS}}$ & $29.21 \pm 1.06^{\mathrm{NS}}$ \\
\hline Sum & $14.38 \pm 4.82^{d}$ & $266.21 \pm 2.67^{b}$ & $247.19 \pm 12.66^{c}$ & $293.41 \pm 11.18^{\mathrm{a}}$ & $296.00 \pm 11.59^{a}$ \\
\hline \multicolumn{6}{|l|}{ Esters } \\
\hline Ethyl acetate & ND & $7.56 \pm 0.59^{b}$ & $8.05 \pm 0.70^{b}$ & $12.93 \pm 0.66^{\mathrm{a}}$ & $13.57 \pm 0.59^{\mathrm{a}}$ \\
\hline Ethyl butanoate & ND & ND & ND & $1.16 \pm 0.08^{\mathrm{NS}}$ & $1.24 \pm 0.12^{\mathrm{NS}}$ \\
\hline Ethyl decanoate & ND & $11.15 \pm 0.62^{c}$ & $14.00 \pm 1.28^{\mathrm{ab}}$ & $14.42 \pm 0.46^{\mathrm{a}}$ & $12.41 \pm 0.87^{\mathrm{bc}}$ \\
\hline Ethyl 9-decenoate & ND & ND & ND & ND & $0.95 \pm 0.10$ \\
\hline Ethyl dodecanoate & ND & $2.12 \pm 0.12^{b}$ & $2.13 \pm 0.07^{b}$ & $2.15 \pm 0.12^{b}$ & $2.40 \pm 0.12^{\mathrm{a}}$ \\
\hline Ethyl heptanoate & ND & $1.93 \pm 0.08^{c}$ & $2.10 \pm 0.10^{b c}$ & $2.76 \pm 0.15^{\mathrm{a}}$ & $2.21 \pm 0.08^{b}$ \\
\hline Ethyl hexanoate & ND & $16.21 \pm 0.41^{c}$ & $12.62 \pm 0.77^{d}$ & $26.45 \pm 0.48^{\mathrm{a}}$ & $18.29 \pm 0.84^{b}$ \\
\hline Ethyl nonanoate & ND & $0.95 \pm 0.04^{b}$ & $1.53 \pm 0.07^{\mathrm{a}}$ & $0.74 \pm 0.07^{c}$ & $0.66 \pm 0.04^{c}$ \\
\hline Ethyl octanoate & ND & $31.13 \pm 1.36^{\mathrm{b}}$ & $39.28 \pm 1.28^{\mathrm{a}}$ & $38.64 \pm 6.19^{a}$ & $32.97 \pm 2.52^{\mathrm{b}}$ \\
\hline Ethyl pentanoate & ND & ND & $0.65 \pm 0.06^{c}$ & $1.32 \pm 0.04^{\mathrm{a}}$ & $1.07 \pm 0.08^{b}$ \\
\hline Isoamyl acetate & ND & $33.27 \pm 4.12^{\mathrm{b}}$ & $33.57 \pm 3.91^{b}$ & $50.40 \pm 5.50^{\mathrm{a}}$ & $45.16 \pm 2.82^{\mathrm{a}}$ \\
\hline Isobutyl acetate & ND & $1.04 \pm 0.09^{c}$ & ND & $3.21 \pm 0.27^{\mathrm{a}}$ & $1.71 \pm 0.03^{b}$ \\
\hline Phenylethyl acetate & ND & ND & ND & $2.26 \pm 0.10^{\mathrm{NS}}$ & $2.36 \pm 0.09^{\mathrm{NS}}$ \\
\hline Isoamyl propionate & ND & ND & ND & $0.64 \pm 0.09$ & ND \\
\hline Sum & ND & $105.35 \pm 5.18^{d}$ & $113.94 \pm 7.35^{c}$ & $157.10 \pm 1.32^{\mathrm{a}}$ & $135.01 \pm 4.58^{b}$ \\
\hline \multicolumn{6}{|l|}{ Miscellaneous } \\
\hline 1, 3, 6-Octatriene, 3, 7-dimethyl-, (E)- & $0.42 \pm 0.32^{c}$ & $6.22 \pm 0.49^{b}$ & $8.72 \pm 0.64^{\mathrm{a}}$ & $8.59 \pm 0.38^{\mathrm{a}}$ & $6.24 \pm 0.63^{b}$ \\
\hline 1, 3, 7-Octatriene, 3, 7-dimethyl-, (E)- & ND & $0.63 \pm 0.04^{\mathrm{NS}}$ & $0.70 \pm 0.11^{\mathrm{NS}}$ & ND & ND \\
\hline 2,3-Butanediol & $0.15 \pm 0.15$ & ND & ND & ND & ND \\
\hline Acetic acid & $0.74 \pm 0.86^{\mathrm{b}}$ & $8.21 \pm 0.19^{\mathrm{a}}$ & $8.13 \pm 0.63^{\mathrm{a}}$ & $8.40 \pm 0.33^{\mathrm{a}}$ & $8.25 \pm 0.17^{\mathrm{a}}$ \\
\hline D-Limonene & ND & ND & ND & $0.64 \pm 0.04$ & ND \\
\hline Linalool & ND & $0.80 \pm 0.08^{\mathrm{NS}}$ & ND & $0.93 \pm 0.07^{\mathrm{NS}}$ & ND \\
\hline Oxime-, methoxy-phenyl- & ND & $1.84 \pm 0.02^{\mathrm{NS}}$ & $1.93 \pm 0.06^{\mathrm{NS}}$ & ND & ND \\
\hline Sum & $1.76 \pm 0.64^{c}$ & $21.66 \pm 0.97^{\mathrm{a}}$ & $22.10 \pm 0.22^{\mathrm{a}}$ & $21.12 \pm 0.64^{\mathrm{a}}$ & $15.82 \pm 0.64^{b}$ \\
\hline Total & $15.69 \pm 4.59^{d}$ & $393.21 \pm 6.81^{c}$ & $383.20 \pm 4.59^{c}$ & $471.64 \pm 12.01^{\mathrm{a}}$ & $446.82 \pm 16.52^{b}$ \\
\hline
\end{tabular}

Values of different superscripts in the same row are significantly different $(p<0.05)$

ND not detected.

aromatic profiles in wine. Phenylalanine, valine, and leucine were utilized and derived 2-phenylethanol, isobutanol, and isoamyl alcohol [26]. Higher alcohols are necessary precursors for the synthesis of some esters, which is desirable as an increased concentration of esters enhances the wine flavor [27].

Esters are one of the most important volatile compounds and are largely responsible for the fruity aroma in wine [26]. A total of 14 esters were identified in golden dried longan wines (Table 2). Among the identified 
esters, isoamyl acetate, ethyl acetate, ethyl decanoate, ethyl hexanoate, and ethyl octanoate were the most prominent esters in these wines, and also contribute to the fruity and floral flavor. Similar research publication was reported by Zhang et al. [7], who found five esters had contents above their thresholds, and the results were also found to be consistent with research on commercial wine, which found that the esters have an effect on the aromatic profiles of the wines [26]. The simultaneous culture led to a higher production of most of the esters compared to the other cultures and exhibited the highest total volatile aroma content (471.64 mg/l), which was in agreement with previous findings [7, 11, 25].

Two major groups of esters are synthesized during fermentation by yeasts: acetate esters and ethyl esters [26]. Acetate esters are formed from an alcohol (complex alcohol derived from amino acid metabolism or ethanol) and an acetyl-CoA [28]. Most acetate esters (isoamyl acetate, ethyl acetate, isobutyl acetate, isoamyl propionate, and phenylethyl acetate) in golden dried longan wine cultures with a co-culture had a higher level than in the monocultures, similar results were observed by Zhang et al. [7] and Arslan et al. [25]. Among the acetate esters, isoamyl acetate (banana note) was detected in the largest amount by the co-culture in wines, in which the yeast was associated with an overexpression of IAH-encoded ester degrading enzyme and related with the branchedchain amino acid transferase gene BAT1, which is correlated with an enhanced production of isoamyl alcohol, which is the precursor of isoamyl acetate [29]. The concentration of alcohol and acetyl-CoA and the activity of the alcohol acetyltransferase (AAT) affect the formation of acetate esters [28]. These acetate esters are important as they are associated with desirable floral and fruity flavors in wine [27].

Among the identified esters, ethyl esters provide a pleasant fruity and floral note and are formed from an alcohol (ethanol) and a short or medium-chain fatty acylCoA derivative via an alcoholysis mechanism. Alcoholysis is a transferase reaction, in which the acyl moiety of acyl-CoA is transferred to an alcohol [30, 31]. The major ethyl esters identified in golden dried longan wines produced from the pure and co-cultures were ethyl decanoate, ethyl hexanoate, and octanoate, with their content ranging from 7 to $50 \mathrm{mg} / \mathrm{l}$. The co-culture presented significantly more higher ethyl esters than the other cultures, possibly due to the fermentation anaerobic conditions, whereby medium chain fatty acyl-CoAs accumulate, which results in increased ethyl ester synthesis [32]. Additionally, the significantly highest concentrations of ethyl nonanoate and ethyl octanoate were in the T. delbrueckii culture. This finding was in agreement with recent researches that reported the concentration of ethyl esters depends on the concentration of the fatty acid precursor [31] and that a significant amount of medium chain (C4-C10) fatty acids accumulate with a T. delbrueckii culture [2].

In addition, others volatiles including terpenes and acids, were also detected in these wines. Terpenes provide the main floral and fruity character of wine and have low odor thresholds [7]. Terpenes, including 1,3,6octatriene, 3,7-dimethyl-, (E)-, 1,3,7-octatriene, 3,7dimethyl-, (E)-, D-limonene, and linalool, were identified in these wines (Table 2). These compounds present in glycoside form can be released by yeast hydrolytic enzymes via acid-induced hydrolysis [33]. Linalool (citrus-like notes) was found in the S. cerevisiae pure culture and the simultaneous culture. Other volatile compounds detected in these wines included acetic acid and oxime-, methoxy-phenyl.

\section{Odor activity values (OAVs) and principal component analysis (PCA)}

The OAVs is a measure of the importance of a specific compound to the odor of a food sample [3]. Volatile compounds with an OAVs higher than 1 provide a pleasant wine aroma [17]. As seen in Table 3, twelve odorants were selected and quantified (Isoamyl alcohol, 2-phenylethanol, ethyl acetate, ethyl butanoate, ethyl decanoate, ethyl hexanoate, ethyl octanoate, isoamyl acetate, isobutyl acetate, phenylethyl acetate, D-limonene and linalool). Ethyl octanoate showed a higher OAVs than the other odorants, which contribute to the floral and fruity odors in a concentration ranging from 15565 to $16485 \mathrm{mg} / \mathrm{l}$. Similar results were observed by Chen and Liu [12] and Chen and Liu [17] in lychee wines, in which they found that ethyl hexanoate and ethyl octanoate were the major compounds in their four treated wines and also that ethyl hexanoate and ethyl octanoate had higher OAVs. The OAVs of ethyl octanoate in the T. delbrueckii culture was the highest, but there was no significant difference with the culture with simultaneous inoculation. 
Table 3. The odor activity values (OAVs) in golden dried longan wines cultured with different yeast strains.

\begin{tabular}{|c|c|c|c|c|c|c|c|}
\hline Compounds & $\begin{array}{l}\text { Longan } \\
\text { juice }\end{array}$ & S. cerevisiae & T. delbrueckii & Simultaneous & Sequential & $\begin{array}{l}\text { Odor Threshold } \\
(\mathrm{mg} / \mathrm{l})\end{array}$ & References \\
\hline \multicolumn{8}{|l|}{ Alcohols } \\
\hline Ethanol & - & - & - & - & - & - & - \\
\hline Ethylhexanol & - & - & - & - & - & - & - \\
\hline Isobutanol & - & 0.26 & 0.19 & 0.54 & 0.51 & 40 & [45] \\
\hline Isoamyl alcohol & - & 2.24 & 2.02 & 3.25 & 3.41 & 30 & [45] \\
\hline Methionol & - & - & - & - & - & - & - \\
\hline 2-Phenylethanol & - & 2.71 & 2.97 & 2.92 & 2.92 & 10 & {$[45]$} \\
\hline \multicolumn{8}{|l|}{ Esters } \\
\hline Ethyl acetate & - & 1.01 & 1.07 & 1.72 & 1.81 & 7.5 & [45] \\
\hline Ethyl butanoate & - & - & - & 58 & 62 & 0.02 & \\
\hline Ethyl decanoate & - & 55.75 & 70.00 & 72.10 & 62.05 & 0.2 & [46] \\
\hline Ethyl 9-decenoate & - & - & - & - & - & - & - \\
\hline Ethyl dodecanoate & - & 0.36 & 0.36 & 0.36 & 0.41 & 5.9 & [45] \\
\hline Ethyl heptanoate & - & - & - & - & - & - & - \\
\hline Ethyl hexanoate & - & 3242.00 & 2524.00 & 5290.00 & 3658.00 & 0.005 & [12] \\
\hline Ethyl nonanoate & - & - & - & - & - & - & - \\
\hline Ethyl octanoate & - & 15565.00 & 19640.00 & 19320.00 & 16485.00 & 0.002 & [45] \\
\hline Ethyl pentanoate & - & - & - & - & - & - & - \\
\hline Isoamyl acetate & - & 1109.00 & 1119.00 & 1680.00 & 1505.33 & 0.03 & [17] \\
\hline Isobutyl acetate & - & 0.65 & - & 2.00 & 1.07 & 1.6 & [46] \\
\hline Phenylethyl acetate & - & - & - & 9.04 & 9.44 & 0.25 & [46] \\
\hline Isoamyl propionate & - & - & - & - & - & - & - \\
\hline \multicolumn{8}{|l|}{ Miscellaneous } \\
\hline 1, 3, 6-Octatriene, 3, 7-dimethyl-, (E)- & - & - & - & - & - & - & - \\
\hline 1, 3, 7-Octatriene, 3, 7-dimethyl-, (E)- & - & - & - & - & - & - & - \\
\hline 2,3-Butanediol & - & - & - & - & - & - & - \\
\hline Acetic acid & - & 0.04 & 0.04 & 0.04 & 0.04 & 200 & [45] \\
\hline D-Limonene & - & - & - & 42.67 & - & 0.015 & [47] \\
\hline Linalool & - & 53.33 & - & 62.00 & - & 0.015 & [45] \\
\hline Oxime-, methoxy-phenyl- & - & - & - & - & - & - & - \\
\hline
\end{tabular}

However, the co-culture presented significantly higher levels of isoamyl alcohol, ethyl acetate, ethyl decanoate, ethyl hexanoate, and isoamyl acetate. These results let us hypothesize that mixed fermentation can improve and enhance the wine quality, as has been confirmed by several researchers, who have reported that co-cultures (i.e., T. delbrueckii and S. cerevisiae) can produce more higher esters, which contribute more to the aroma in wine.

PCA was applied to assess the differences between different fermentations and these aromatic compounds.
The first two principal components accounted for $83.67 \%$ of the total variance in the wines, with $\mathrm{PC} 1$ accounting for 72.94\% of the total variance and PC2 27.06\% (Fig. 1). The wines obtained from both pure and mixed cultures could be clearly separated, possibly due to the different formation paths of the aroma compounds in these wines (Fig. 1A). The simultaneous and sequential cultures were in the positive part of PC1, and there was high levels of alcohol and esters in these wines, which were correlated to the high concentration of OAVs in the cocultures (Table 3). This finding was in agreement with 
A
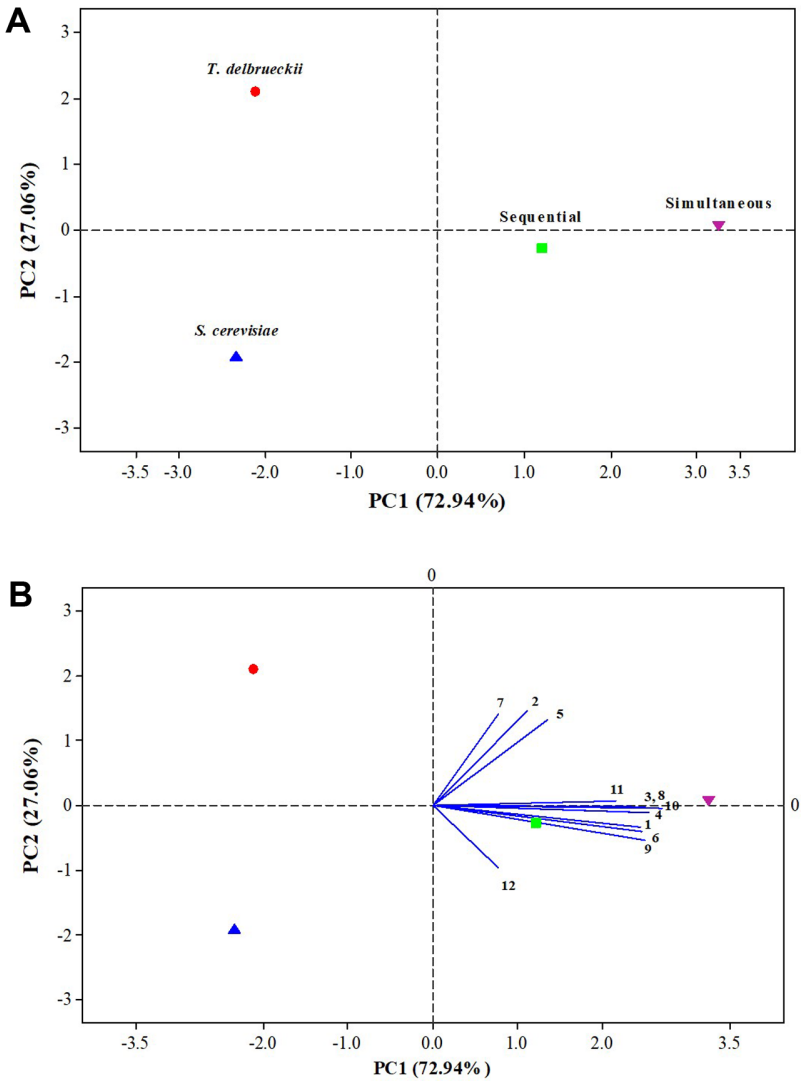

Fig. 1. The principal component analysis for selected volatiles in golden dried longan wines fermented with four cultures. Score plot of the yeast strains (A) Biplot between the yeast strains and volatile compounds (B): Isoamyl alcohol (1); 2Phenylethanol (2); Ethyl acetate (3); Ethyl butanoate (4); Ethyl decanoate (5); Ethyl hexanoate (6); Ethyl octanoate (7); Isoamyl acetate (8); Isobutyl acetate (9); Phenylethyl acetate (10); D-Limonene (11); Linalool (12).

recent research demonstrating that the co-culture fermentation of $T$. delbrueckii and S. cerevisiae generated remarkably more of the desired aroma compounds [7]. The biplot of Isoamyl alcohol, ethyl acetate, ethyl butanoate, ethyl hexanoate, isoamyl acetate, isobutyl acetate, phenylethyl acetate and D-limonene were positioned in the positive part of $\mathrm{PC} 1$ and were also found between both the co-culture plots, suggesting that these volatile compounds could be produced at a high level from both co-cultures (Fig. 1B). Furthermore, the biplot of 2-phenylethanol, ethyl octanoate, and ethyl decanoate were located between the T. delbrueckii plot and the simultaneous culture plot in the positive part of PC2, which could explain why their odors obtained from the T. delbrueckii and the simultaneous culture showed higher values. Additionally, linalool was found in the positive part of $\mathrm{PC} 1$ and the negative part of $\mathrm{PC} 2$, which were also positioned between the sequential culture plot and S. cerevisiae.

\section{Total phenolic content and antioxidant activity}

It is well accepted that wine is rich in oxidants. Several researchers have reported that wine phenolics have health-protective properties, such as against diabetes, osteoporosis, obesity, allergies, neurodegenerative diseases, cardiovascular diseases, and some cancers [34]. As a result, the total phenolic content of golden dried longan juice was significantly higher than for the other wines (17.807 $\mathrm{mg} \mathrm{GAE} / \mathrm{ml}$ ), followed by the sequential, simultaneous, S. cerevisiae, and T. delbrueckii cultures, with values of 5.80, 5.34, 5.20, and $4.95 \mathrm{mg} / \mathrm{ml}$, respectively (Table 4). These results were higher than those reported in red wine, which varied from $1.207-4.263 \mathrm{mg} /$ $\mathrm{ml}$, while only a few phenolics were found in fresh longan wine $(0.781 \mathrm{mg} / \mathrm{ml})$ [11]. Furthermore, the total phenolic content of the juice was about three-fold higher than for the other wines. Similarly, Kelebek and Selli [35] found that the total phenolic content of mandarin juice was two-fold higher than for the other wines, possibly due to

Table 4. The total phenolic content and the antioxidant activity of golden dried longan juice and wines.

\begin{tabular}{lcccc}
\hline & $\begin{array}{c}\text { Total phenolics } \\
(\mathrm{mg} \mathrm{GAE} / \mathrm{ml})\end{array}$ & $\begin{array}{c}\text { DPPH } \\
(\mathrm{mg} \mathrm{TEAC} / \mathrm{ml})\end{array}$ & $\begin{array}{c}\text { ABTS } \\
(\mathrm{mg} \mathrm{TEAC} / \mathrm{ml})\end{array}$ & $\begin{array}{c}\text { FRAP } \\
(\mathrm{mg} \mathrm{TEAC} / \mathrm{ml})\end{array}$ \\
\hline Juice & $17.807 \pm 0.535^{\mathrm{a}}$ & $11.180 \pm 0.169^{\mathrm{a}}$ & $47.314 \pm 1.844^{\mathrm{a}}$ & $8.788 \pm 0.371^{\mathrm{a}}$ \\
S. cerevisiae & $5.20 \pm 0.19^{\mathrm{c}}$ & $4.43 \pm 0.10^{\mathrm{d}}$ & $24.54 \pm 0.23^{\mathrm{c}}$ & $5.14 \pm 0.40^{\mathrm{c}}$ \\
T. delbrueckii & $4.95 \pm 0.12^{\mathrm{c}}$ & $4.49 \pm 0.09^{\mathrm{d}}$ & $23.53 \pm 0.39^{\mathrm{c}}$ & $5.12 \pm 0.13^{\mathrm{c}}$ \\
Simultaneous & $5.34 \pm 0.10^{\mathrm{bc}}$ & $4.98 \pm 0.10^{\mathrm{c}}$ & $24.97 \pm 0.35^{\mathrm{c}}$ & $5.26 \pm 0.21^{\mathrm{c}}$ \\
Sequential & $5.80 \pm 0.16^{\mathrm{b}}$ & $6.80 \pm 0.23^{\mathrm{b}}$ & $28.06 \pm 0.38^{\mathrm{b}}$ & $5.97 \pm 0.22^{\mathrm{b}}$ \\
\hline
\end{tabular}

Values of different superscripts in the same column are significantly different $(p<0.05)$ 
the transformation of phenolic compounds into condensed forms that possessed slightly different chemical properties, as was also confirmed by Akalin et al. [36] in pomegranate juice and wines.

The antioxidant capacity of golden dried longan wines was estimated by three methods namely, DPPH, ABTS, and FRAP. DPPH is an assay method that measures changes in the concentration of stable free radicals according to the electron-donating ability of the sample [37]. The oxidation activity of the wines ranged from 4.43 to $6.80 \mathrm{mg} \mathrm{TEAC} / \mathrm{ml}$, and $11.18 \mathrm{mg} \mathrm{TEAC} / \mathrm{ml}$ for the juice. Here, the golden dried longan wines had a markedly higher antioxidant capacity (DPPH) than reported in the previous studies of Kelebek and Selli [35] and Lorenzo et al. [38].

The antioxidant capacity values of the wines from different cultures decreased in the order: golden dried longan juice $>$ sequential $>$ simultaneous $>T$. delbrueckii $>$ S. cerevisiae. The decrease in the antioxidant capacity values of dried longan juice was possibly due to the formation and oxidation of phenolic compounds [35].

In ABTS, a radical cation is synthesized in the stable form using potassium persulphate [39]. The antioxidant activity of the wines by the ABTS assay ranged from 23.53-28.06 mg TEAC/ml. Among the four treatments, the sequential culture had the highest antioxidant capacity, followed by the simultaneous, S. cerevisiae, and T. delbrueckii cultures. However, the antioxidant capacity of dried longan juice was higher than for the wines, confirming earlier findings [35].

The FRAP assay determines the reducing potential of an antioxidant reacting with an $\mathrm{Fe}^{3+}$-TPTZ complex to producing $\mathrm{Fe}^{2+}$-TPTZ [39]. The FRAP values ranged from a minimum of 5.12 to a maximum of $8.79 \mathrm{mg}$ $\mathrm{TEAC} / \mathrm{ml}$ in wines fermented with $T$. delbrueckii and dried longan juice, respectively. Dried longan wine prepared by the sequential culture between $S$. cerevisiae and T. delbrueckii had the highest FRAP value $(5.97 \mathrm{mg}$ TEAC/ml) among the four fermented.

The Pearson's correlation coefficients of total phenolic content with the DPPH, FRAP, and ABTS assays were $0.954,0.992$, and 0.974 , respectively. The differences among the three antioxidant activity assays, namely the DPPH, ABTS, and FRAP, results depended on the chemical reagent involved. However, these results also showed a strong correlation between total phenolic con- tent and the three methods used for testing the antioxidant capacity, confirming that phenolic compounds may affect the radical scavenging activity of golden dried longan juice and wine, corroborating the results in the wines tested by other researchers [16, 35, 38].

Amino acids are synergistic antioxidants. Their mechanism can be enhanced by the chelation of pro-oxidative metal traces and by regeneration of the oxidized primary antioxidants [40]. Additionally, it was previously reported that proline functions as an antioxidant, and also it was suggested it scavenges intracellular reactive oxygen species (ROS) to protect cells from oxidative damage [41]. $\beta$-Alanine functions (at least in part) by combining with histidine to generate carnosine by the enzyme carnosine synthase, in which carnosine is a non-enzymatic free radical scavenger and natural antioxidant [42, 43]. Furthermore, other researchers have demonstrated that GABA is produced primarily by the decarboxylation of L-glutamic acid and catalyzed by glutamate decarboxylase and also that it functions as a bioactive compound. This bioactive compound could potentially protect against or ameliorate the oxidative stress induced by nephrectomy or chronic kidney disease [44]. As seen in Table 1, proline, alanine, glutamic acid, leucine, and GABA were identified as the main amino acids in golden dried longan wines. These results let us hypothesize that the residues of amino acids, especially the most abundant amino acids, are correlated to the total phenolic content and related to the antioxidant activity in golden dried longan juice and wines.

\section{Acknowledgments}

This research was financially supported by National Pingtung University of Science and Technology.

\section{Conflict of Interest}

The authors have no financial conflicts of interest to declare.

\section{References}

1. Chang CY, Chang CH, Yu TH, Lin LY, Yen YH. 1998. The effect of drying treatment on the flavour and quality of longan fruit. Food Flavours: Formation Analysis and Packaging Influences 40: 353367.

2. Azzolini M, Fedrizzi B, Finato F, Vagnoli P, Scrinzi C. 2012. Effects of 
Torulaspora delbrueckii and Saccharomyces cerevisiae mixed cultures on fermentation and aroma of Amarone wine. Eur. Food Res. Technol. 235: 303-313.

3. Azzolini M, Tosi E, Lorenzini M, Finato F, Zapparoli G. 2015. Contribution to the aroma of white wines by controlled Torulaspora delbrueckii cultures in association with Saccharomyces cerevisiae. World J. Microbiol. Biotechnol. 31: 277-293.

4. Renault P, Miot-Sertier C, Marullo P, Hernández-Orte P, Lagarrigue L, Lonvaud-Funel A, et al. 2009. Genetic characterization and phenotypic variability in Torulaspora delbrueckii species: Potential applications in the wine industry. Int. J. Food Microbiol. 134: 201-210.

5. Hansen EH, Nissen P, Sommer P, Nielsen JC, Arneborg N. 2001. The effect of oxygen on the survival of non-Saccharomyces yeasts during mixed culture fermentations of grape juice with Saccharomyces cerevisiae. J. Appl. Microbiol. 91: 541-547.

6. Renault P, Coulon J, Barbe JC, Bely M. 2015. Increase of fruity aroma during mixed T.delbrueckii/S.cerevisiae wine fermentation is linked to specific esters enhancement. Int. J. Food Microbiol. 207: 40-48.

7. Zhang BQ, Luan Y, Duan CQ, Yan GL. 2018. Use of Torulaspora delbrueckii Co-fermentation with two Saccharomyces cerevisiae strains with different aromatic characteristic to improve the diversity of red wine aroma profile. Front. Microbiol. 9: 606.

8. Surin $S$, Thakeow $P$, Seesuriyachan $P$, Angeli $S$, Phimolsiripol $Y$. 2014. Effect of extraction and concentration processes on properties of longan syrup. J. Food Sci. Technol. 51: 2062-2069.

9. Trinh TTT, Woon WY, Yu B, Liu SQ. 2010. Effect of L-isoleucine and L-phenylalanine addition on aroma compound formation during longan juice fermentation by a co-culture of Saccharomyces cerevisiae and Williopsis saturnus. S. Afr. J. Enol. Vitic. 31: 116-124.

10. Trinh TTT, Yu B, Curran P, Liu SQ. 2012. Formation of aroma compounds during longan juice fermentation by Williopsis saturnus var. saturnus with the addition of selected amino acids. J. Food Process Preserv. 36: 198-206.

11. Liu G, Sun J, He X, Tang Y, Li J, Ling D, et al. 2018. Fermentation process optimization and chemical constituent analysis on longan (Dimocarpus longan Lour.) wine. Food Chem. 256: 268-279.

12. Chen D, Liu SQ. 2014. Chemical and volatile composition of lychee wines fermented with four commercial Saccharomyces cerevisiae yeast strains. Int. J. Food Sci. Tech. 49: 521-530.

13. Waters C. 1993. Waters AccQ. Tag Chemistry Package (Instruction Manual), pp. 1-5. Ed. Millipore Corporation, Milford, MA, USA.

14. Zeng F, Ou J, Huang Y, Li Q, Xu G, Liu Z, et al. 2015. Determination of 21 free amino acids in fruit juices by HPLC using a modification of the 6-aminoquinolyl-N-hydroxysuccinimidyl carbamate (AQC) method. Food Anal. Methods 8: 428-437.

15. Nuengchamnong N, Ingkaninan K. 2010. On-line HPLC-MS$\mathrm{DPPH}$ assay for the analysis of phenolic antioxidant compounds in fruit wine: Antidesma thwaitesianum Muell. Food Chem. 118: 147-152.

16. Buyuktuncel E, Porgalı E, Colak C. 2014. Comparison of total phenolic content and total antioxidant activity in local red wines determined by spectrophotometric methods. Food Nutr. Sci. 5: 1660-1667.

17. Chen D, Liu SQ. 2016. Impact of simultaneous and sequential fermentation with Torulaspora delbrueckii and Saccharomyces cerevisiae on non-volatiles and volatiles of lychee wines. LWT-Food Sci. Technol. 65: 53-61.

18. Ingledew WM, Magnus CA, Sosulski FW. 1987. Influence of oxygen on proline utilization during the wine fermentation. Am. J. Enol. Vitic. 38: 246-248.

19. Brandriss MC, Falvey DA. 1992. Proline Biosynthesis in Saccharomyces cerevisiae: Analysis of the $P R O 3$ gene, which encodes $\Delta^{1}$ Pyrroline-5-carboxylate reductase. J. Bacteriol. 174: 3782-3788.

20. Tomenchok DM, Brandriss MC. 1987. Gene-enzyme relationships in the proline biosynthetic pathway of Saccharomyces cerevisiae. J. Bacteriol. 169: 5364-5372.

21. Brandriss MC. 1983. Proline utilization in Saccharomyces cerevisiae: Analysis of the cloned PUT2 gene. Mol. Cell. Biol. 3: 1846-1856.

22. Takagi H. 2008. Proline as a stress protectant in yeast: physiological functions, metabolic regulations, and biotechnological applications. Appl. Microbiol. Biotechnol. 81: 211-223.

23. Degols G, Jauniaux JC, Wiame JM. 1987. Molecular characterization of transposable-element-associated mutations that lead to constitutive L-ornithine aminotransferase expression in Saccharomyces cerevisiae. Eur. J. Biochem. 165: 289-296.

24. Sumrada RA, Cooper TG. 1984. Nucleotide sequence of the Saccharomyces cerevisiae arginase gene (CAR1) and Its transcription under various physiological conditions. J. Bacteriol. 160: 1078-1087.

25. Arslan E, Celik ZD, Cabaroglu T. 2018. Effects of pure and mixed autochthonous Torulaspora delbrueckii and Saccharomyces cerevisiae on fermentation and volatile compounds of narince wines. Foods 7: 1-12.

26. Swiegers JH, Bartowsky EJ, Henschke PA, Pretorius IS. 2005. Yeast and bacterial modulation of wine aroma and flavour. Aust. J. Grape Wine Res. 11: 139-173.

27. Lee PR, Ong YL, Yu B, Curran P, Liu SQ. 2010. Evolution of volatile compounds in papaya wine fermented with three Willopsis saturnus yeasts. Int. J. Food Sci. Techol. 45: 2032-2041.

28. Zhang J, Zhang C, Qi Y, Dai L, Ma H, Guo X, et al. 2014. Acetate ester production by Chinese yellow rice wine yeast overexpressing the alcohol acetyltransferase-encoding gene ATF2. Genet. Mol. Res. 4: 9735-9746.

29. Lilly M, Bauer FF, Lambrechts MG, Swiegers JH, Cozzolino D, Pretorius IS. 2006. The effect of increased yeast alcohol acetyltransferase and esterase activity on the flavour profiles of wine and distillates. Yeast 23: 641-659.

30. Saerens SMG, Delvaux FR, Verstrepen KJ, Thevelein JM. 2010. Production and biological function of volatile esters in Saccharomyces cerevisiae. Microbe. Biotechnol. 3: 165-177.

31. Saerens SMG, Verstrepen KJ, Van Laere SDM, Voret ARD, Dijck PV, Delvaux FR, et al. 2005. The Saccharomyces cerevisiae EHT1 and EEB1 genes encode novel enzymes with medium-chain fatty acid ethyl ester synthesis and hydrolysis capacity. J. Biol. Chem. 281: 4446-4456. 
32. Äyräpää T, Lindström I. 1977. Aspects of the influence of exogenous fatty acids on the fatty acid metabolism of yeast. Proc. Eur. Brew Conv. 16: 507-517.

33. Moreno-Arribas MV, Polo M. 2009. Wine Chemistry and Biochemistry. pp. 463-496. Ed. Springer, New York.

34. Fernandes I, Pérez-Gregorio R, Soares S, Mateus N, Freitas V. 2017. Wine flavonoids in health and disease prevention. Molecules 22: 292.

35. Kelebek H, Selli S. 2014. Identification of phenolic compositions and the antioxidant capacity of mandarin juices and wines. J. Food Sci. Technol. 51: 1094-1101.

36. Akalin AC, Bayram M, Anli RE. 2017. Antioxidant phenolic compounds of pomegranate wines produced by different maceration methods. J. Inst. Brew. 124: 38-44.

37. Rahman MM, Islam MB, Biswas M, Khurshid Alam AH. 2015. In vitro antioxidant and free radical scavenging activity of Nardostachys jatamansi DC. J. Acupunct. Meridian Stud. 3: 112-118.

38. Lorenzo CD, Badea M, Colombo F, Orgiu F, Frigerio F, Pastor RF, et al. 2017. Antioxidant activity of wine assessed by different in vitro methods. BIO Web of Conferences. 9: 1-6.

39. Rajurkar NS, Hande SM. 2011. Estimation of phytochemical content and antioxidant activity of some selected traditional indian medicinal plants. Ind. J. Pharm. Sci. 73: 146-151.

40. Marcuse R. 1960. Antioxidative effect of amino-acids. Nature 186: 886-887.
41. Takagi $\mathrm{H}$, Taguchi J, Kaino T. 2016. Proline accumulation protects Saccharomyces cerevisiae cells in stationary phasefrom ethanol stress by reducing reactive oxygen specie. Yeast 33: 355-363.

42. Mocchegiani E, Straub RH. 2004. Possible new anti-ageing strategies related to neuroendocrine-immune interactions. Neurolmmune Biol. 4: 399-407.

43. Schnuck JK, Sunderland KL, Kuennen MR, Vaughan RA. 2016. Characterization of the metabolic effect of $\beta$-alanine on markers of oxidative metabolism and mitochondrial biogenesis in skeletal muscle. J. Exerc. Nutr. Biochem. 20: 34-41.

44. Diana M, Quílez J, Rafecas M. 2014. Gamma-aminobutyric acid as a bioactive compound in foods: a review. J. Funct. Foods 10: 407420.

45. Guth H. 1997. Quantitation and sensory studies of character impact odorants of different white wine varieties. J. Agric. Food Chem. 45: 3027-3032.

46. Cullere L, Escudero A, Cacho J, Ferreira V. 2004. Gas chromatographyolfactometry and chemical quantitative study of the aroma of six premium quality spanish aged red wines. J. Agric. Food Chem. 52: 1653-1660.

47. Casassa LF, Sari SE, Bolcato EA, Diaz-Sambueza MA, Catania AA, Fanzone ML, et al. 2019. Chemical and sensory effects of cold soak, whole cluster fermentation, and stem additions in pinot noir wines. Am. J. Enol. Vitic. 70: 19-33. 